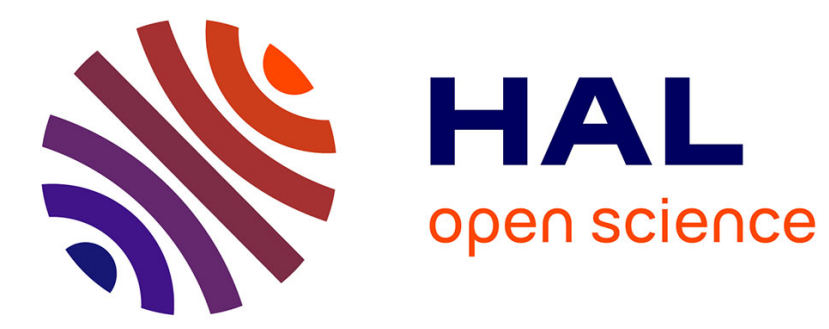

\title{
GeNova04 : la fabrique d'un nouveau patrimoine identitaire à Gênes
}

Maria Elena Buslacchi

\section{To cite this version:}

Maria Elena Buslacchi. GeNova04 : la fabrique d'un nouveau patrimoine identitaire à Gênes. Culture et Musées, 2019, 33, pp.61-86. 10.4000/culturemusees.2845 . hal-03254221

\section{HAL Id: hal-03254221 \\ https://hal.science/hal-03254221}

Submitted on 8 Jun 2021

HAL is a multi-disciplinary open access archive for the deposit and dissemination of scientific research documents, whether they are published or not. The documents may come from teaching and research institutions in France or abroad, or from public or private research centers.
L'archive ouverte pluridisciplinaire HAL, est destinée au dépôt et à la diffusion de documents scientifiques de niveau recherche, publiés ou non, émanant des établissements d'enseignement et de recherche français ou étrangers, des laboratoires publics ou privés. 


\title{
OpenEdition
}

Journals

\section{Culture \& Musées}

Muséologie et recherches sur la culture

$33 \mid 2019$

La fabrique des patrimoines européens au XXIe siècle

Dossier

\section{GeNova04 : la fabrique d'un nouveau patrimoine identitaire à Gênes}

\author{
GeNova04: Building a New Heritage Identity in Genoa
}

GeNova04: la fabricación de un nuevo patrimonio de identidad en Génova

\section{Maria Elena Buslacchi}

p. $61-86$

https://doi.org/10.4000/culturemusees.2845

\section{Résumés}

Français English Español

Cet article, issu de l'expérience de terrain conduit pendant une thèse doctorale en anthropologie sociale et historique, interroge la fabrique du patrimoine culturel génois par les élites locales de la fin du XXe et du début XXIe siècle. Il étudie la redécouverte d'un héritage oublié, légitimant la recherche d'une reconnaissance européenne et internationale. En particulier, l'analyse s'intéresse aux dynamiques culturelles et urbaines produites autour du label Capitale européenne de la culture en 2004 à Gênes comme moment majeur de l'objectivation culturelle (Handler, 1988) d'une identité génoise, inscrit dans un processus de patrimonialisation (Palumbo, 2003 ; Davallon, 2006) de plus longue durée.

This paper presents the case-study of a patrimonialization process in the context of a Genoese cultural heritage, staged by local elites as a forgotten heritage rediscovered, in a bid to gain European and international recognition. The paper focuses on the 2004 European Capital of Culture event, identified as a key moment of the cultural objectivation (Handler, 1988) of a Genoese identity, within a longer process of patrimonialization (Palumbo, 2003; Davallon, 2006).

En este artículo, basado en la experiencia de campo realizada durante mi tesis en antropología social e histórica, presenta el caso de la fábrica de un patrimonio cultural genovés, establecido por las élites locales a finales del siglo XX - principios del XXI - como el redescubrimiento de un legado olvidado que justificó la búsqueda de reconocimiento europeo e internacional. En particular, mi análisis se enfoca en el evento de la Capital Europea de la Cultura en 2004 como un momento importante en la objetivación cultural (Handler, 1988) de una identidad genovesa, insertada en el proceso de patrimonialización (Palumbo, 2003; Davallon, 2006) a largo plazo. 


\section{Entrées d'index}

Mots-clés : Capitales européennes de la culture, patrimonialisation, tourisme patrimonial, politiques culturelles

Keywords: European Capitals of Culture, patrimonialization, heritage tourism, cultural policies Palabras clave: Capitales Europeas de la Cultura, desarrollo del patrimonio, turismo patrimonial, políticas culturales

Notes de la rédaction

Manuscrit reçu le 20 décembre 2018

Version révisée reçue le 16 octobre 2019

Article accepté pour publication le 24 octobre 2019

\section{Texte intégral}

1 Dans cet article, je propose d'observer comment plusieurs logiques de fabrique du patrimoine peuvent évoluer et s'imbriquer autour d'un même objet, en décrivant l'effet d'une politique culturelle européenne sur la configuration des acteurs et des techniques de la patrimonialisation. La politique que j'étudie est celle des Capitales européennes de la culture (CEC), et le cas d'étude concerne la ville de Gênes, labellisée en 2004. Dans un premier temps, je vais décrire comment la politique européenne de la CEC intervient, entre la fin des années 1990 et le début des années 2000, dans un contexte de transformation urbaine de plus longue durée. Il s'agira d'observer les dispositifs mis en place pour l'application de cette politique culturelle européenne au niveau local et leur imbrication avec les processus déjà à l'œuvre. Ces dispositifs de médiation, d'implication de la société civile et de communication marquent le passage d'une logique savante de la patrimonialisation à une logique identitaire qui met l'accent sur la dimension internationale et, en particulier, européenne de l'histoire de la ville. Une fois les techniques d'étude présentées, j'essaierai d'en saisir l'impact à moyen et long terme.

2 Comme on le verra à travers la reconstruction historique des phases en amont et en aval de l'événement, la définition de certains critères comme éléments identitaires, au détriment d'autres, est stratégiquement utilisée par ses promoteurs ainsi que certains acteurs « avertis » pour introduire de nouvelles formes de gouvernance urbaine et en tirer une conséquente reconfiguration des pouvoirs locaux. En effet, si l'identité industrielle de la ville avait commencé à être mise à l'écart des représentations officielles depuis la fin des années 1980, ce n'est qu'avec la labellisation que le processus de transformation urbaine déjà à l'œuvre assume une véritable dimension identitaire, dont les marqueurs font l'objet de négociations entre les groupes sociaux. Par le biais d'une méthodologie de gouvernance nouvelle, importée à Gênes depuis Barcelone ${ }^{1}$, une écriture collective et, pourrait-on dire, sociale du patrimoine est lancée, et ce moment initie la forme mythifiée, voire sacralisée, d'une nouvelle fondation de la ville, comme le slogan GeNovao4 en témoigne.

3 Pour décrire ce moment et mettre en perspective les dynamiques qui l'ont caractérisé, je m'appuie sur mon travail de thèse qui avait pour objectif de retracer, en croisant des approches historique anthropologique et sociologique, les phases d'application et de travail d'une politique culturelle européenne à l'échelle urbaine. Le travail se fonde sur l'analyse comparée de plusieurs sources d'information. En premier lieu, les sources d'archives : articles de presse, littérature dite " grise » concernant la candidature, la production et la programmation de la CEC 2004, documents préparatoires, dossiers, délibérations officielles, transcriptions des conseils municipaux, matériaux de communication et comptes rendus produits à l'issue de l'événement, qui m'ont permis de reconstruire les faits, les étapes et d'identifier un premier panel de témoins à rencontrer. En deuxième lieu, ma recherche s'est nourrie des entretiens que j'ai réalisés entre 2012 et 2015 avec les opérateurs culturels, les politiques, les membres d'associations et collectifs impliqués dans la CEC. Au-delà du premier panel d'interviewés, j'ai collecté les témoignages d'autres personnes ayant joué un rôle souvent moins public dans le processus, mais que j'avais identifiées en croisant les 
récits ou en étudiant les documents. En troisième lieu, je me suis servie pour mon analyse des données quantitatives produites par les collectivités locales et publiques, des organismes de recherche (par exemple l'Istat) ou académiques (Université de Gênes, Université Bocconi) depuis la période en question, telles que les chiffres de fréquentation des lieux (avant, pendant, après la labellisation), des enquêtes de satisfaction, des études d'impact économique. Ma recherche se développant entre dix et quinze ans après les phénomènes étudiés, ces sources sont utilisées de manière comparative avec le présent pour cerner l'impact à moyen et long terme de l'événement. La production d'enquêtes quantitatives n'ayant pas été homogène dans les vingt dernières années, ni dans la périodicité ni dans la forme, ce travail réunit une hétérogénéité de données où l'approche qualitative se révèle essentielle à la compréhension. L'observation de près m'a permis d'analyser cette évolution de manière critique, en restituant par une démarche ethnographique la complexité des formes du réel sans qu'elles soient aplanies sur un modèle univoque, ni confondues dans leurs prémisses théoriques, et témoigne des parcours multiples, pas nécessairement linéaires, par lesquels la fabrique des patrimoines peut être questionnée.

\section{Le label de "Capitale européenne de la culture » (CEC) : un processus urbain à long terme}

$4 \quad$ Le parcours de transformation symbolique et matérielle de la ville de Gênes (Italie) commence dans les années 1980 par une action volontaire de la Mairie. Cela se produit en réaction à la constitution d'un " problème public » (Cefaï, $1996: 48$ ) autour de la situation économique, architecturale et environnementale de la ville qui, selon le discours des interventionnistes, avait sacrifié son paysage à un développement industriel qui ne garantissait plus sa contrepartie en termes d'emplois. Dépourvue, donc, selon ce récit, à la fois de beauté et d'emplois, la ville était considérée comme en retard par rapport à d'autres territoires : une " coalition de croissance » (Molotch, 1976) s'organise dès lors pour pousser les collectivités à mettre en route des actions de transformation. Dans la définition, négociée et souvent conflictuelle du chemin à emprunter pour qu'un changement se produise ${ }^{2}$, deux pôles émergent : la redécouverte d'un héritage artistique oublié et l'usage des formes architecturales contemporaines comme moteurs d'une économie fondée sur le tourisme et l'attractivité du territoire. Ces nouveaux centres d'intérêt sont issus de la confrontation avec les expériences d'autres villes européennes et nord-américaines ; le milieu universitaire, les anciennes familles marchandes et la bourgeoisie entrepreneuriale contribuent largement à nourrir ce discours par leurs voyages, par les relations tissées et la mobilisation de cas observés dans d'autres pays. Comme l'architecte Renzo Piano, qui travaille au même temps à la réalisation du Centre Pompidou à Paris et à la réhabilitation du Vieux Port (Porto Antico) à Gênes à la fin des années 1980, plusieurs professionnels contribuent à pointer comment l'héritage historique de la ville pourrait être mis en valeur par l'insertion dans le paysage urbain de nouveaux éléments d'attraction, en évoquant des précédents jugés réussis. Claudio Burlando, jeune élu au conseil municipal et futur maire de Gênes (1992-1993), avait eu l'idée de construire un aquarium sur le modèle de celui de Baltimore, qu'il avait pu visiter en 1987 lors d'un voyage institutionnel à l'occasion de l'attribution d'un doctorat honoris causa au spécialiste de Christophe Colomb, Paolo Emilio Taviani. L'ingénieur Andrea Rossi, directeur du Porto Antico, prenait la reconversion du front de mer de Baltimore comme modèle d'action et en avait mobilisé de manière directe les professionnels (par exemple Peter Chermaieff pour l'aménagement de l'aquarium, aux côtés de Renzo Piano). L'universitaire Bruno Gabrielli, adjoint à l'urbanisme et au centre historique de la Mairie de Gênes entre 1997 et 2001, et ensuite, de 2001 à 2006, à la qualité urbaine et aux politiques culturelles, avait travaillé comme consultant du maire de Barcelone Pasqual Maragall i Mira et proposait d'introduire à Gênes son système de gouvernance locale pour le 
développement d'une nouvelle « idée de ville » fondée sur le tourisme patrimonial. Dans ce contexte, Glasgow ou Bilbao constituaient aussi des repères urbains récurrents dans le discours public de l'époque.

La socialisation de ce nouveau regard sur la ville inaugurera la patrimonialisation d'une "identité génoise » dans une perspective internationale. Jean Davallon (2006), pour définir cette première phase du processus, évoque le concept de "trouvaille " d'Umberto Eco (1993), où quelque chose de commun et d'ordinaire se fait soudainement objet d'intérêt collectif et déclenche des " gestes » conséquents. J'ai donc choisi de suivre ce paradigme des gestes pour décrire les itinéraires de patrimonialisation qui se déroulent à Gênes à compter des années 1980, à la fois en parallèle et en intersection. Ainsi, par la confrontation avec d'autres expériences, un centre historique négligé depuis les dommages de la Seconde Guerre mondiale et des friches industrielles et portuaires désormais improductives deviennent à Gênes le fondement d'une stratégie d'action institutionnelle qui, par sa portée sociale, mérite d'être analysée à partir d'une perspective ethnographique et théorique (Abélès 1983, 1990, 1992 ; Abélès \& Jeudi, 1997). Le potentiel de la récupération du passé et des injonctions à la modernité est présenté comme la clef d'un progrès urbain « naturel », sur lequel la ville de Gênes était dite en retard. Dans ce cadre d'inspiration à des références étrangères, décrites comme l'expression d'un standard à atteindre rapidement 3 , sous pression des élites, l'administration municipale a recours à un outil qui va accélérer les actions de transformation urbaine : le grand événement (Roche, 2000 ; Guala, 2002). En effet, l'Exposition internationale spécialisée de 1992, consacrée aux explorations géographiques et en particulier au navigateur Christophe Colomb, est suggérée par Renzo Piano et aboutit à la reconversion du Vieux Port en nouvel espace public, aux formes architecturales inédites et à la fonction ludique et attractive. Le Sommet du G8 en 2001 au Palazzo Ducale entraîne de nombreuses restaurations du tissu historique et montre la ville sur les écrans du monde entier. Ces événements préparent assurément le terrain pour la mise en place de la CEC ; ils permettent à la fois aux collectivités locales d'accéder à des sources de financement extraordinaires pour la réalisation de grands travaux et de les présenter comme une marque de reconnaissance internationale. Ce repositionnement de la ville est en effet ratifié par la considération du Bureau international des Expositions et l'adhésion des pays étrangers à l'Expo 1992, ainsi que, pour le Sommet du G8 de 2001, par le protocole international et ses annexes médiatiques. Si cet article se focalise sur GeNovao4, la portée et le sens de l'événement ne pourraient pas se comprendre sans leur insertion dans ce cadre d'évolution de plus longue durée.

\section{La candidature au label « Ville européenne de la culture », entre logiques savantes et logiques sociales}

L'observation de l'application de la politique de la CEC au niveau local dans cette perspective historique permet de saisir le rôle que le label a joué dans la fabrique d'un patrimoine culturel génois. La première candidature au label «Ville européenne de la culture » (ce n'est qu'en 1998 que la mention devient "Capitale ») avait été présentée par la Ville en juin 1997 sur la suggestion du président de la Porto Antico SpA, société de gestion des nouveaux espaces réaménagés pour l'Exposition de 1992, dénommée Expo 1992. Comme le rappelle Chito Guala, sociologue et adjoint au maire, qui avait cordonné le dossier, « tout le monde » avait pris part à la candidature, c'est-à-dire toute personne comptant dans le secteur culturel et entrepreneurial en ville, soit les élites qui avaient déjà dirigé les premières actions de transformation urbaine. Parmi eux, naturellement, Renzo Piano, mais aussi Nicola et Bacci Costa, à la tête de l'Aquarium, ou les auteurs d'études en histoire de l'art sur les résidences des familles nobles génoises et leurs collections au fil des siècles ${ }^{4}$. Le choix des axes principaux de la candidature découle assez naturellement des travaux en cours et repose sur les « trouvailles » identifiées par ces élites comme potentiel à développer, que je qualifie de 
premier geste de la patrimonialisation. La candidature coïncide avec une mise en cohérence des savoirs qui avaient été produits par les universitaires sur le passé historique de la ville, notamment sur sa dimension d'État, de République, de port et de pôle d'échanges commerciaux, culturels, artistiques sur une échelle territoriale très étendue (deuxième geste de la patrimonialisation). L'usage stratégique de ce savoir dans le développement d'un intérêt touristique potentiel est aussi mis en avant, et légitimé à la fois par la comparaison à l'international et par son attestation académique5. Le thème du voyage devient donc central : déjà utilisé pour l'Expo 1992, il sera repris comme fil rouge de la programmation de la CEC.

$7 \quad$ L'accent patrimonial est porté sur des périodes précises de l'histoire de la ville, avec une attention particulière à l'époque baroque, et sur le rôle de la République de Gênes dans l'échiquier géographique euro-méditerranéen (le recours à la mention "Capitale de la mer » est commun). Ces choix traduisent l'effort d'affirmer à l'époque contemporaine un nouveau " positionnement international » de la ville en se servant d'une logique savante. Au même moment, des études préparatoires à la candidature Unesco des Palazzi dei Rolli sont présentées comme « le premier exemple en Europe » de planification urbanistique conçue par les autorités administratives avec un plan unitaire répondant à des mesures spécifiques d'organisation et de représentation diplomatique (Poleggi, 1998). Illustrées par Pieter-Paul Rubens lors d'un séjour à Gênes au début du XVIIe siècle ${ }^{6}$, les quarante-deux résidences en question (qui seront effectivement inscrites au patrimoine mondial de l'Unesco en 2006) font partie d'un ensemble plus large de demeures nobiliaires bâties entre le XVIe et le XVIIe siècle par les représentants de l'oligarchie génoise afin de montrer leur pouvoir et leur richesse à leurs alliés et rivaux, tant locaux qu'étrangers. Ces résidences étaient inscrites dans des registres singuliers qui les classaient selon leur degré de splendeur et les habilitaient à recevoir un certain type d'invités d'honneur (ducs, comtes, princes, rois, empereurs, etc.) par un tirage au sort qui chargeait la famille propriétaire de l'accueil des visiteurs officiels dans la République de Gênes. De même, la candidature mobilise l'histoire de la circulation d'artistes et des œuvres liées au mécénat de familles nobles telles que les Grimaldi, Costa, Lomellini, Pallavicini, Durazzo, Fieschi, Doria, Brignole, De Ferrari, ainsi que les relations qui les unissaient, au fil des siècles, aux plus puissantes maisons européennes. Cette focalisation ciblée de manière instrumentale est une première étape de la filiation inversée (Pouillon, 1975, 1977 ; Lenclud, 1987 ; Davallon, 2000) qui fera privilégier, dans le récit fondateur de GeNovao4, les références à ces moments de l'histoire locale sur tout le reste : le comité auteur de la candidature reconstruit, depuis le présent, un passé et des ancêtres mythiques pour établir un rapport de descendance directe avec le présent.

La candidature de Gênes (1997) n'aboutit pas pour l'année 2001, où les villes de Rotterdam et Porto sont choisies comme CEC, mais un changement des modalités d'assignation du titre fait en sorte que les autres villes candidates sont nommées CEC pour les années suivantes : Bruges et Salamanque en 2002, Graz en 2003, Lille et Gênes en 2004, et ainsi de suite jusqu'en 2010. Le 28 mai 1998, les auteurs de la candidature génoise fêtent donc la nomination, les ressources financières qui seront bientôt disponibles et la confirmation du succès de la stratégie de transformation urbaine entamée7. Le dossier de candidature définit ainsi le périmètre de ce qui sera considéré comme « culture » de la ville et mis en valeur en 2004 (troisième geste de la patrimonialisation). Le maire Adriano Sansa venait d'achever son mandat, en novembre 1997, de même que les adjoints qui avaient géré avec lui l'après 1992. Son successeur, Giuseppe Pericu, avait donc la tâche de préparer l'événement, qui est symboliquement associé à une stratégie de développement de la ville dans son ensemble.

\section{L'enjeu identitaire : jeux d'acteurs, reconfiguration des pouvoirs locaux et participation citoyenne}


9 Lorsque la préparation à la CEC se met en place, le pari sur la culture comme choix stratégique de développement urbain a déjà été fait par la Mairie, poussée par les élites locales. Les frontières de cette culture ont été esquissées de manière assez détaillée par les auteurs du dossier de candidature. Par exemple, la Mairie dispose déjà d'une production savante de connaissances sur l'héritage architectural et artistique, fournie par l'université et mise en perspective selon une logique sociale. Ce n'est qu'alors, pourtant, que la CEC devient un instrument majeur de production d'identité à l'échelle urbaine. Pour le nouveau maire, le but prioritaire du travail engagé en vue des deux grands événements imminents (le G8 est aussi prévu en 2001) est la récupération d'une fierté, d'un sentiment d'appartenance considéré comme «oublié » ou «perdu ${ }^{8}$. La définition des contenus détaillés de la programmation de la CEC passe alors par la mise en œuvre institutionnelle d'un projet collectif qui attribue à l'événement le pouvoir de re-fonder symboliquement la ville. L'année 2004 est proposée comme l'horizon sacré d'affichage d'une transformation urbaine aboutie grâce à la valorisation d'un nouveau patrimoine local, qui se veut ouvert à tous à partir des axes déjà identifiés. Cette logique identitaire, par ses modes de fonctionnement rituels et son discours sacralisant, dépasse la définition savante du patrimoine aussi bien que celle - sociale - des élites marchandes (Rautenberg, 2003a) et propose aux habitants de s'approprier cette stratégie urbaine comme « foi urbaine » dans un projet porté par les élites et comme opportunité pour l'avenir. Le grand événement est alors pour le maire, mais aussi pour certains de ses adjoints, fonctionnaires et consultants associés au projet CEC, l'occasion d'opérer une « objectivation culturelle » (Handler, 1988) de ce que l'on entendra par "Gênes » dans le futur, objectivation issue d'une négociation contrôlée entre les acteurs. Convié à participer non seulement à la programmation, mais aussi à la conception de l'année CEC comme marque de changement, chacun est libre de se projeter en tant qu'opérateur de la transformation, c'est-à-dire comme un protagoniste de la nouvelle Gênes. Et cela, en ayant recours à un capital symbolique déjà construit, tiré essentiellement du passé historique de la ville, à vénérer et incarner comme une identité génoise à travers le temps. À cette fin, la phase préparatoire de la CEC marque l'organisation institutionnelle de l'accès du collectif à un objet patrimonial reconstitué (quatrième geste de patrimonialisation) grâce à plusieurs parcours parallèles : tout d'abord, la constitution de dispositifs de médiation du patrimoine, dont font partie des expositions majeures au Palazzo Ducale et la systématisation d'une scène muséale ; puis, une socialisation de l'enjeu de la transformation urbaine par l'association de la société civile aux actions culturelles ; enfin, une politique de communication de plus en plus puissante, centrée sur l'affichage du changement. Ces techniques finiront par s'imbriquer au fur et à mesure que les travaux avanceront en vue de l'année 2004, plusieurs acteurs acceptant de jouer le rôle de témoin de ce changement, soit par calcul stratégique, soit par conviction sincère.

\section{La mise en place des dispositifs d'accès au patrimoine culturel}

La production de connaissances déjà disponibles est rendue accessible au grand public par des actions de vulgarisation des recherches et de médiation du patrimoine, sous la direction d'historiens de l'art qui associent une activité académique à un engagement dans les institutions culturelles urbaines ${ }^{9}$. En premier lieu, nous pouvons mentionner les expositions Van Dyck a Genova (1997) ou El Siglo de los Genoveses (1999-2000), organisées au Palazzo Ducale pour raconter les liens des familles génoises en Europe du XVIe au XVIIIe siècle. Notamment la première qui, à travers le récit de la présence dans la ville, de 1621 à 1627, du peintre Anton van Dyck (Anvers, 1599 - Londres, 1641), raconte au public un moment où, à Gênes, les collectionneurs et les mécènes attiraient les plus importants artistes et œuvres de leurs époques. L'aménagement de la salle d'ouverture fait expérimenter aux visiteurs l'ambiance d'une chambre de collectionneur génois au XVIIe siècle, où l'on pourrait trouver à la fois des œuvres de Rubens, Caravage, Simon Vouet, Bernardo Strozzi, Titien, Tintoret. Le public 
découvre aussi que ces ouvres sont aujourd'hui hébergées aux National Gallery de Washington, de Londres et d'Édimbourg, au Louvre et au Prado, dans les Musées capitolins : le fait que le contexte génois ait joué un rôle important dans leur production est constamment mis en avant. Ces expositions, proposées par Palazzo Ducale SpA, sont explicitement conçues comme un parcours unitaire à l'horizon de 2004, comme leurs descriptifs officiels le déclarent. L'alliance entre l'entreprise publique de gestion de l'institution culturelle naissante et la Mairie se fera ici en étroite collaboration, au point que le rôle de Palazzo Ducale SpA se superposera souvent à celui de la Direction culturelle de la Ville dans les années suivantes. Les actions de l'année CEC seront programmées par l'entreprise Genova $2004 \mathrm{SrL}$, à laquelle participent Palazzo Ducale SpA pour la Mairie de Gênes, l'agence Filse pour la Région Liguria, le Port, l'université et la chambre de commerce. De même, l'internationalisation déjà décrite est confiée à Enrico Da Molo, ancien fonctionnaire auprès de la Commission européenne, qui est chargé de mettre en pratique les indications des comités, issues des groupes de travail. La dimension européenne de l'action est donc réaffirmée par le choix des protagonistes dans sa gestion et sa construction ${ }^{10}$. Les artistes convoqués, en revanche, demeurent pour la plupart locaux et italiens, issus d'un concours qui retient environ 120 projets artistiques et 70 colloques et rencontres scientifiques parmi 850 propositions ${ }^{11}$. À la différence des autres CEC qui suivront, ce n'est pas tant un patrimoine culturel et artistique venant d'ailleurs en Europe que les Génois vont rencontrer, que la mise en lumière de leur propre patrimoine. Cet adjectif possessif est souvent répété dans la communication institutionnelle, qui essaie ainsi d'induire le développement d'un sentiment d'appropriation et d'appartenance chez les habitants.

En vue de 2004, la réorganisation des musées municipaux participe à améliorer la connaissance et la lisibilité de la «nouvelle » ville. Une étude commanditée en 2000 par la ville de Gênes à la société Roland \& Berger identifie trois pôles : les Musées de Strada Nuova - la Nouvelle Voie ouverte en 1550 dans l'ancien tissu urbain médiéval, où se trouvent les Palazzi Rosso, Bianco et Tursi avec leurs galeries -, le pôle moderne dans le quartier oriental de Nervi et, enfin, le pôle maritime, constitué du Musée de la mer et de la navigation ${ }^{12}$ à développer avec le projet de réaménagement de la Darse et le Musée naval de Pegli. Ces pôles correspondent aux axes de programmation de la CEC, à savoir Ville d'art, Capitale de la mer, Ville contemporaine (Città d'Arte, Capitale del Mare, Città Contemporanea), et à ces axes correspondent aussi les trois expositions majeures organisées au Palazzo Ducale en 2004 : L'Età di Rubens, I transatlantici et Arti e architetture. Bien que la valorisation du passé industriel ne soit pas complètement oubliée ${ }^{13}$, dans ce contexte la production culturelle se concentre en premier lieu sur la « ville d'art » et notamment sur la ville baroque. Le Palazzo Tursi héberge l'exposition L'Invenzione dei Rolli. Genova, civiltà di palazzi, qui explique le contenu de la candidature au patrimoine de l'Unesco. L'exposition L'Età di Rubens. Dimore, committenti e collezionisti genovesi au Palazzo Ducale revient une fois de plus sur les rapports entre la République de Gênes et le reste de l'Europe. Comme dans l'exposition sur van Dyck, l'aménagement est conçu pour donner l'illusion au public d'entrer dans les demeures d'une quinzaine de collectionneurs et mécènes génois. La proposition invite aussi à explorer les quadrerie (galeries d'exposition) de deux Palazzi dei Rolli, la Galerie nationale du Palazzo Spinola et le Musée du Palazzo Rosso, et assigne à l'exposition une valeur d'expérience subjective, pensée pour toucher émotionnellement le visiteur, qui devrait en sortir émerveillé... ou fier, sentiment présenté comme éminemment génois ${ }^{14}$.

\section{GeNova04 : une écriture collective et communicationnelle de la culture}

12 Cette fierté locale se place au centre des objectifs du projet génois de la CEC, car elle est censée être alimentée par une autre approche : l'implication des habitants dans le projet. Ce point sera présent de manière récurrente, surtout au cours des années suivantes dans les recommandations des commissions d'évaluation des candidatures au 
label CEC. À Gênes, au début, cette implication ne se fait pas par injonction des institutions européennes, mais, encore une fois, par émulation d'une autre expérience européenne. La méthode de la conférence stratégique avait été proposée au maire par son adjoint Bruno Gabrielli, qui l'avait importée de Barcelone. L'objectif était de réunir autour d'une table tous les porteurs d'intérêt dans les différents domaines pour partager une réflexion sur les perspectives de développement de la ville au-delà de la durée des mandats électoraux. Le caractère rituel de cet acte est évident et contribue à la «sacralisation » du processus. Les travaux, initiés en 1999, durent plusieurs mois et transforment de manière assez importante le pari sur la culture qui avait été fait une dizaine d'années auparavant en une véritable ligne stratégique d'action à long terme, où tout sujet aurait un rôle à jouer. La démarche de changement et de repositionnement à l'échelle internationale passe ainsi d'une question exclusive des élites et des experts vers un enjeu identitaire majeur : un effort commun de transformation urbaine où tout acteur citoyen a sa part de responsabilité. La fabrique du patrimoine local dans une perspective européenne y est déjà centrale. L'outil est nouveau, et la participation citoyenne ne l'est pas moins ${ }^{15}$, ce qui ne manque pas de susciter un certain scepticisme parmi les conviés. L'affirmation du centre historique comme un patrimoine culturel à mettre en valeur en est la clef stratégique réaffirmée : ainsi, certains espaces et bâtiments, comme la place De Ferrari ou le Palais ducal, sont élus quartiers généraux de la mise en scène de la nouvelle ville et, en même temps, acquièrent une importance symbolique pour les opérateurs culturels génois, qui contribueront dans les années suivantes à les animer.

Sur ce mode de fonctionnement, un groupe de travail de personnes provenant de différents parcours politiques et culturels est constitué en vue de l'année CEC 2004 et prend le nom de Tavolo regionale permanente della cultura. Son but est d'élaborer des propositions et des demandes aux collectivités en matière de culture, selon une procédure participative inspirée de celle de la conférence stratégique. Directeurs de théâtres, de compagnies, de salles de musique, de galeries d'art, de festivals, d'associations culturelles en font partie : être présents et actifs signifiait acquérir une place sur la scène culturelle locale pour la suite. Ces instances culturelles pour 2004 sont ainsi résumées par Salvatore Vento, membre de groupe de travail et auteur du volume Genova 2004. In viaggio con le associazioni :

« [Nous demandons] un engagement à soutenir et renforcer 1) la ville en tant que
patrimoine [“bene culturale” en italien, N.D.A.], par la valorisation du centre
historique et de l'environnement, la combinaison de la culture et du tourisme, le
support à la transformation de la ville et l'aide à son ouverture culturelle ; 2) la
communication, comme méthodologie et comme projet qui accompagne la
naissance des nouvelles activités et professions, pour collaborer avec les
opérateurs du domaine de la culture, pour exporter et importer des expériences
significatives en Italie et à l'étranger, et pour dialoguer avec les professionnels,
activer des réseaux et se coordonner ; 3) une organisation managériale au service
de la ville, pour mettre en lien le public et le privé, pour insérer à des niveaux de
direction des professionnels d'expertise notoire, pour considérer les expériences
des autres et pour un usage plus rationnel des espaces pour les événements - les
musées, Palazzo Ducale, Porto Antico... » (Vento, 2004 : 105).

Ces instances, à la suite du processus de négociation adopté, ont fini par s'aligner sur celles de la Mairie. Elles sont assumées par plusieurs opérateurs culturels qui, dans la perspective de 2004, se font à leur tour les auteurs d'une multiplication d'actes de production de savoir (deuxième étape) et de production culturelle (quatrième étape). Leurs actions reproduisent à nouveau, mais « d'en bas », la même filiation inversée qui avait été opérée par les auteurs de la candidature. En échange des engagements demandés, le groupe de travail proposait à la Ville, entre autres, le développement de projets culturels en cohérence avec les axes thématiques. Ces projets vont constituer le noyau de la programmation culturelle de la ville dans les années à venir, et leurs auteurs seront mentionnés ensuite par différents acteurs du service Culture de la Mairie comme une « guilde » qui ne pourra plus dorénavant être exclue de la mise en place d’initiatives institutionnelles dans les secteurs d'expertise qui la concernent. 
15 Par ailleurs, en vue de l'année GeNovao4, la communication apparaît comme un enjeu majeur de la politique de la ville, avec des ressources financières dédiées et un public ciblé, tant au niveau local qu'à l'international ${ }^{16}$.

16 Le conseil municipal adopte en juillet 2000 un changement iconique dans le blason de la ville. Un détail graphique qui semble revêtir une dimension politique et symbolique de premier plan : les queues jusqu'alors tombantes des griffons qui portent l'emblème de Gênes sont retournées vers le haut. Ce travail sur l'image acquiert de plus en plus d'importance, et c'est à ce moment que la Mairie se dote d'experts en marketing territorial, encore peu consultés avant cette date. Si les premiers objectifs étaient de « sortir la ville de l'obscurité » et faire connaitre Gênes « autrement que comme la ville à côté de Portofino ", au cours de la Conférence stratégique de 1999 l'idée de " fierté " et d'une " identité » à développer se fait centrale. En vue de GeNova04, une nouvelle adjointe à la promotion de la ville, Anna Castellano, est nommée en 2002. Formée à la London School of Economics, à Londres, elle sera en même temps déléguée à l'administration de Palazzo Ducale SpA et mandatée sur les missions de gestion économique, de coordination de projet et de marketing. Elle explique qu'à cette occasion, pour la première fois, la Mairie se dote de plans stratégiques pour la communication et la promotion du territoire, en collaboration avec la province et malgré l'absence d'une stratégie à l'échelle régionale (Comune di Genova, 2005 : 12). Avec elle, un Tavolo di promozione della città est constitué en 2002 par des représentants de la Mairie, de la Province, de la chambre de commerce, puis élargi en 2003 à l'agence de promotion touristique régionale en Ligurie pour commencer un travail de standardisation et d'homogénéisation de l'image urbaine. Les premières interventions se traduisent en une opération de coordination des activités existantes afin d'éviter les superpositions non souhaitées et de rendre le calendrier des initiatives plus lisible, à la fois par les habitants et par les touristes. À cette époque se discutent des labels comme "Genova estate " pour rassembler les activités estivales ou "Paganiniana » pour le prix et les concerts dédiés à Niccolò Paganini, le célèbre violoniste et compositeur génois : des étiquettes communicationnelles qui traduisent l'offre développée par les différents opérateurs culturels en un message cohérent, qu'ils sont invités à partager. Par ailleurs, le logo élaboré pour l'année de la Capitale européenne de la culture, Ge-Nova, marque explicitement l'idée de changement par la juxtaposition de l'abréviation «Ge », pour Gênes ${ }^{17}$, et de l'adjectif « Nova », " nouvelle » en latin et très proche de l'italien nuova. Cette déclaration de changement est donc matérialisée sur différents supports, drapeaux, autocollants, étendards, etc., distribués aux commerçants pour qu'ils les affichent dans leurs magasins, en plus d'être exposés dans les lieux institutionnels, comme la façade du Palazzo Ducale ${ }^{18}$. Certains de ces exemplaires ont résisté dans l'espace public jusqu'en 2019.

\section{Quels bilans autour de cette nouvelle identité urbaine?}

Bien des ressources ont été exploitées par la Mairie, ou ses bras opérationnels, pour que le travail mené en vue de l'année 2004 produise une "substantialisation » de la nouvelle identité de la ville (Palumbo, 2003) et se stabilise dans l'opinion publique, en assurant la pérennité et l'efficacité de ce changement d'image. Les premiers effets sont inscrits dans le paysage : les nouvelles formes architecturales, héritage de 1992 ou développées en lien avec le Musée de la mer et les grands travaux publics de réaménagement pour le Sommet du G8 sont mis en avant dans le discours public comme éléments catalyseurs d'une nouvelle attractivité. La stratégie politique consistant à attribuer constamment une valeur historique, artistique et culturelle à l'héritage de la vieille ville, en misant dessus comme une ressource, plutôt que comme un objet de soins, finit par rassembler les intérêts et l'attention de plusieurs acteurs. En effet, une partie des habitants accepte de contribuer activement à la « requalification du centre » en profitant des incitations mises en place par la Mairie pour restaurer la 
qualité de l'habitat. Les Palazzi dei Rolli seront, par exemple, inscrits en 2006 sur la liste du patrimoine de l'Unesco.

D'autres indicateurs peuvent, peut-être, nous en dire davantage sur les effets immédiats au niveau socio-économique : l'ancien maire Giuseppe Pericu évoque en entretien l'ouverture de certains hôtels gérés par des chaînes internationales ou les prix de l'immobilier en hausse. Les fonctionnaires du service Culture citent le nombre de visites dans les musées et de nuitées dans les hôtels. Sur un temps plus long, les chiffres de fréquentation des musées ${ }^{19}$ et du Palazzo Ducale ${ }^{20}$ marquent un intérêt renouvelé à la fois local, national et international pour les collections, les expositions temporaires et l'offre culturelle proposée. L'exposition L'Età di Rubens avait attiré 195000 visiteurs et se plaçait en seconde position dans le classement des expositions les plus visitées en Italie en 2004.

Pourtant, en ce qui concerne l'appropriation de l'élément identitaire, le seul sondage d'opinion conduit à la suite de la CEC est une enquête quantitative sur le changement de la perception que les Italiens ont de la ville de Gênes, réalisée en 2005. Ce travail avait été confié à Demoskopea, l'un des sponsors principaux de la manifestation, dont le rôle demeure évidemment ambigu. Selon cette enquête, à la question « Est-ce que la Capitale européenne de la culture a produit un changement pour la ville de Gênes ? ", la réponse est positive pour $72,3 \%$ des Italiens et $88,5 \%$ des Génois. Aux yeux de 87,8 \% des Italiens, ce changement serait certainement ou probablement pérenne ; la confiance est plus importante dans le cas des Génois (91,1\%); et $48 \%$ des habitants se disaient plus fiers de leur ville après 2004, tandis que 50 \% n'avaient pas changé d'opinion et $2 \%$ choisissaient de ne pas répondre.

Mon analyse ne visant pas à juger du succès ou de l'insuccès de la CEC, ces déclarations et ces données demeurent au final peu significatives. Il est en revanche intéressant, pour acquérir d'autres éléments d'analyse, de suivre les traces de certains protagonistes de GeNova04. Cela peut préciser la manière dont cette proposition d'identité nouvelle a interrogé la reconfiguration des pouvoirs locaux, notamment le poids de la culture dans les politiques urbaines et sur l'adoption de cette attitude entrepreneuriale à l'échelle européenne. Par exemple, l’ingénieur Davide Viziano, qui avait assumé en 2002 la direction de l'entreprise Genova 2004, sera promu la même année à la tête du Conservatoire Niccolò Paganini, qu'il dirigera jusqu'en 2014, tout en restant aussi président de son entreprise familiale historique de BTP Gruppo Viziano. Une consultante pour la Mairie sur la CEC entre 2000 et 2005, et responsable du secteur du spectacle, littérature, cinéma et des rapports avec Lille 2004, sera aussi consultante pour la candidature de Marseille-Provence à la CEC 2013 et travaillera à Gênes au développement du célèbre festival Suq. L'équipe de la Direction de la culture de la Mairie, celle de Palazzo Ducale SpA (depuis 2008 Fondation), du nouveau Musée de la mer, du Porto Antico et de l'Aquarium mettront en place des actions de collaboration et de programmation commune qui donneront vie à une offre culturelle renouvelée : c'est le cas, par exemple, du Festival della Scienza, manifestation créée en 2003 et consacrée à la culture scientifique, qui touchera 250 ooo visiteurs pour les éditions 2006, 2007 et 2008, et investira tous ces lieux simultanément. De même, le Palazzo Ducale, par l'offre constante de grandes expositions payantes à l'envergure internationale ${ }^{21}$, soutiendra une programmation quotidienne d'événements, qui, comme celle de la Ville, se fonde aussi sur la contribution des opérateurs culturels de l'ancien Tavolo per la cultura. Ces exemples nous permettent d'entrevoir les mouvements, de surface et de structure, dans les " mondes de l'art » (Becker, 1988) existants ou émergents en 2004. Si l'alliance entre les opérateurs culturels, les institutions et les collectivités s'inscrit dans la durée, cela sclérosera aussi la scène culturelle locale. Qui n'était pas là en 2004 et n'avait pas participé à cette définition de la nouvelle identité génoise fondée sur la culture aura un accès très difficile à la scène publique et officielle. La plupart des activités de toute une nouvelle génération d'opérateurs demeureront longtemps reléguées dans des espaces mineurs, souvent privés ou associatifs ${ }^{22}$.

21 En conclusion, parmi les retombées les plus intéressantes de la Capitale européenne de la culture à Gênes, j'en retiendrai essentiellement deux : en premier lieu, la mise en place de dynamiques d'équipe inter-institutionnelles qui élargissent les arènes de 
pouvoir dans la gouvernance locale (Pinson, 2003) et développent l'apprentissage commun de nouvelles règles, notamment d'une démarche administrative par projet ; en deuxième lieu, la stabilisation et la substantialisation dans le discours public d'une notion d'identité urbaine (Palumbo, $2003: 32$ ) qui, dorénavant, sera prise en compte par les politiques, les médias, les experts sans plus être sérieusement remise en question. Cette sacralisation marque aussi l'oubli de la logique sociale et identitaire du patrimoine qui avait été adoptée pour développer chez les Génois une fierté de leur ville, en faveur du retour à une logique qui se limite à la préservation de l'héritage dans l'avenir et fait essentiellement l'objet du travail des experts. Les actions municipales suivantes seront alors prioritairement orientées à l'accroissement du tourisme patrimonial (Saidi, 2010) et non plus à cette dimension identitaire, donnée désormais pour acquise.

\section{Bibliographie}

Abélès (Marc). 1983. Les Lieux du politique. Paris : Société d'ethnographie.

Abélès (Marc). 1990. Anthropologie de l'État. Paris : Armand Colin.

Abélès (Marc). 1992. La Vie quotidienne au Parlement européen. Paris : Hachette.

Abélès (Marc) \& Jeudy (Henri-Pierre). 1997. Anthropologie du politique. Paris : Armand Colin.

Becker (Howard S.). Les Mondes de l'art. Trad. de l'anglais de Jeanne Bouniort. Paris : Flammarion (Champs, Arts) [éd. originale : 1982].

Bourdieu (Pierre). 1992. Les Règles de l'art : Genèse et structure du champ littéraire. Paris : Seuil.

Buslacchi (Maria Elena). 2016. Capitales de quelle culture ? L'impact social des politiques culturelles. Thèse de doctorat, EHESS - Centre Norbert Elias et Université de Gênes, soutenue le 21 janvier 2016.

Cefaï (Daniel). 1996. « La construction des problèmes publics. Définitions de situations dans des arènes publiques ». Réseaux, 14(75), p. 43-66.

DOI : $10.3406 /$ reso.1996.3684

Chastel (André) \& Babelon (Jean-Pierre). 2004. La Notion de patrimoine. Paris : Liana Levi.

Davallon (Jean). 2002. « Comment se fabrique le patrimoine? ». Sciences humaines. Hors-série, 36, p. 74-77.

Davallon (Jean). 2006. Le Don du patrimoine. Une approche communicationnelle de la patrimonialisation, Paris : Hermes Science-Lavoisier.

Eco (Umberto). 1993. « Observations sur la notion de gisement culturel ». Traverses, 5, p. 9-18.

Guala (Alessandro Chito). 2002. « Per una tipologia dei mega-eventi ». Bollettino della Società Geografica Italiana, série XII, VII(4), p. 861-894.

Handler (Richard). 1988. Nationalism and the Politics of Culture in Quebec. Madison (WI) : The University of Wisconsin Press.

Lenclud (Gérard). 1987. « La tradition n’est plus ce qu'elle était ». Terrain, 9, p. 110-123.

DOI : $10.4000 /$ terrain.3195

Maione (Valeria). 1996. "Osservazioni in margine alla rilevazione statistica sui visitatori della mostra "Van Dyck a Genova, Grande pittura e collezionismo" ». Bollettino dei Musei civici genovesi, 52-53-54.

Molotch (Harvey). 1976. " The city as a growth machine: toward a political economy of place ». The American Journal of Sociology, 82(2), p. 309-332.

DOI : 10.1086/226311

Palumbo (Berardino). 2003. L'Unesco e il Campanile. Antropologia, politica e beni culturali in Sicilia orientale. Rome : Meltemi.

Pinson (Gilles). 2003. « Le chantier de recherche de la gouvernance urbaine et la question de la production des savoirs dans et pour l'action ». Lien social et politiques, 50, p. 39-55.

Poleggi (Ennio). 1998. Atlante dei palazzi di Genova. Turin : Umberto Allemandi \& C.

Pouillon (Jean). 1975. Fétiches sans fétichisme. Paris : Maspero.

Pouillon (Jean). 1977. "Plus ça change, plus c'est la même chose ». Nouvelle Revue de psychanalyse, 15, p. 203-211.

Rautenberg (Michel). 2003. "Comment s'inventent de nouveaux patrimoines. Usages sociaux, pratiques institutionnelles et politiques publiques en Savoie ». Culture \& Musées, 1, p. 19-40.

DOI : $10.3406 /$ pumus.2003.1165 
Roche (Maurice). 2000. Mega-Events and Modernity: Olympics and Expos in the Growth of Global Culture. Londres : Routledge.

Saidi (Habib). 2010. « De la culture touristique au tourisme patrimonial ». Ethnologies, 32(2), p. 5-22.

DOI : 10.7202/1006303ar

Vento (Salvatore). 2004. In viaggio con le associazioni. Gênes : De Ferrari.

\section{Sources et documents}

Comune di Genova. 1958. Centro storico di Genova: preliminari allo studio del piano di valorizzazione, conservazione e risanamento, relazione del Gruppo di studio presieduto dall'assessore ai LL.PP. Matteo Vita. Gênes : Comune di Genova.

Comune di Genova. 1988. Genova verso il 1992. Gênes : Comune di Genova.

Comune di Genova. 1990. Genova verso il 20oo. Gênes : Comune di Genova.

Comune di Genova. 1999. Genova, le vie del Mediterraneo all'Europa [projet de candidature pour Genova, Città europea della cultura 2004]. Gênes : Comune di Genova.

Comune di Genova. 2004. Proposal for the inscription of Genoa Le Strade Nuove and the System of the Palazzi dei Rolli in the Unesco World Heritage List. Vol. 3 Site Management Plan. Gênes : Comune di Genova.

Comune di Genova. 2005a. Atti del convegno Genova 2004-2006. Un bilancio per il futuro (Genova, Palazzo Ducale, 16 maggio 2005). Gênes : Comune di Genova.

Comune di Genova. 2005b. GeNovao4. Un viaggio lungo un anno. Milan : Skira.

Comune di Genova. 2006. «Il vento del Sud-Est. Regionalismo, neosicilianismo e politiche del patrimonio nella Sicilia di inizio millennio ». Antropologia. Il patrimonio culturale, 6-7, p. 43-91.

\section{Notes}

1 Comme l'on verra plus loin dans ce texte.

2 Ces conflits entre les partisans d'un nouveau développement industriel et ceux d'un développement touristique (davantage que culturel ou créatif, comme ce sera le cas plus tard) caractérisent une première phase de la réflexion collective sur l'identité de la ville, que je ne vais pas traiter ici car ils ne sont plus pertinents à l'époque de la CEC, mais que j'ai analysés dans ma thèse (Buslacchi, 2016).

3 En particulier, parmi d'autres, Bilbao pour le rôle des équipements culturels comme levier de régénération urbaine, Baltimore et Barcelone pour l'aménagement du front de mer, Glasgow (qui avait été CEC en 1990) pour le potentiel de développement associé à un grand événement culturel. Ces villes sont traitées comme termes de comparaison dans des allocutions publiques, des interviews de presse, et même des expositions dédiées (par exemple, Bilbao a Genova. La cultura cambia le città, au Palazzo Ducale en 2003, célébrait la manière dont le Musée Guggenheim avait entraîné une transformation urbaine majeure et en exposait des œuvres en prêt).

4 Ennio Poleggi et le Département d'architecture d'un côté, Ezia Gavazza, Lauro Magnani, Pietro Boccardo, Clario Di Fabio et le Département d'histoire de l'art de l'autre.

5 Par exemple, le Département d'économie et de gestion avait conduit une étude de public lors de l'exposition Van Dyck a Genova (Maione, 1996).

6 Pieter-Paul Rubens, I palazzi di Genova, Anvers, 1622.

7 La subvention initiale est de 150 millions d'euros, auxquels s'ajoutent environ 50 millions de ressources privées. Les dépenses totales pour les interventions structurelles de GeNovaO4 sont d'environ 200 millions d'euros, plus 30 pour les événements et les manifestations (Gastaldi, 2005 ; Guala, 2004, 2007).

8 Entretien avec l'ancien maire de la ville (1997-2007), Giuseppe Pericu, janvier 2013.

9 Par exemple, Piero Boccardo, formé à l'Université de Gênes, l’Universidad Autónoma de Madrid, l'Université de Milan et le Musée du Louvre, était commissaire général de la Galleria di Palazzo Rosso et, depuis 2005, assumera la direction des Musées di Strada Nuova ; Clario di Fabio, formé entre Gênes et Florence, était depuis 1988 commissaire général de la Galleria di Palazzo Bianco et, depuis 1994, du Musée d'architecture et sculpture de Sant'Agostino, et il sera enseignant-chercheur statutaire à l'Université de Gênes à partir de 2005.

10 Voir aussi, dans les lignes qui suivent, le recours à l'architecte espagnol Guillermo Vázquez Consuegra pour le projet du Musée de la mer.

11 Comune di Genova (2005b). 
12 Le Galata - MuMa est aujourd'hui le plus visité de la région et fut aménagé en vue de 2004 dans des espaces de la Darse de la ville récupérés et modernisés par l'architecte Guillermo Vázquez Consuegra. Le nouveau musée contribuait aussi, par des formes architecturales inédites, à crédibiliser l'axe "Ville contemporaine ».

13 Parmi les actions de valorisation du passé industriel, on peut citer l'éclairage nocturne et les panneaux d'explication des grues du Vieux Port. Cet axe a été davantage développé dans les années suivantes : par exemple, depuis 2012, le Galata Open Air Museum expose en plein air les vestiges de l'activité portuaire à la vieille Darse par des grues, des voies, des plateformes roulantes...

14 L'épithète traditionnel de la ville est « la superbe », qui lui aurait été attribué par l'humaniste François Pétrarque (1304-1374).

15 D’autant que la Convention de Faro de 2005 est encore loin d'être rédigée.

16 Les investissements en communication (7 millions d'euros contre les 22 millions dépensés pour la production des manifestations [Comune di Genova, 2005b]) servent à consolider l'effort pour la production d'une nouvelle image de la ville, qui trouve en effet bien plus de crédit auprès de la presse : entre décembre 2003 et décembre 2004, 10531 articles sont publiés sur la Capitale européenne de la culture : en moyenne, donc, une trentaine par jour, dont plusieurs dans la presse étrangère. La société Genova 2004 SrL obtient le grand prix di Pubblicità Italia pour la meilleure campagne dans la section « communication publique », et la Mairie se voit attribuer le Globe Award for the Best New Tourism Project WorldWide in 2004 par le British Guild of Travel Writers.

17 Immédiatement compréhensible en Italie car utilisée, par exemple, dans les adresses postales ou sur les plaques d'immatriculation automobiles.

18 L’identité locale substantialisée a déjà ici pris des formes marchandisées et commercialisées (Palumbo, 2003).

19 Sources : Annuario Statistico del Comune di Genova 2001-2018 et Indagini sulla qualite percepita dai visitatori - Servizi museali gestiti in concessione anno 2016, Cooperativa Solidarietà e Lavoro, 2016.

20 Source : Annuario Statistico del Comune di Genova 2001-2018 et Bilancio sociale de la Fondation de Palazzo Ducale, 2008-2017.

21 On peut évoquer en particulier les propositions issues de la collaboration avec la société Linea d'Ombra et le commissaire Marco Goldin, parmi lesquelles on signalera l'exposition Van Gogh e il viaggio di Gauguin, qui attire 350 ooo visiteurs en 2012.

22 Au Palazzo Ducale, un « quota » est défini : l'espace (souterrain) de la Sala Dogana est explicitement dédié à la créativité des jeunes.

\section{Pour citer cet article}

Référence papier

Maria Elena Buslacchi, « GeNova04 : la fabrique d'un nouveau patrimoine identitaire à

Gênes », Culture \& Musées, 33 | 2019, 61-86.

Référence électronique

Maria Elena Buslacchi, « GeNova04 : la fabrique d'un nouveau patrimoine identitaire à

Gênes ॥, Culture \& Musées [En ligne], 33 | 2019, mis en ligne le 19 novembre 2019, consulté le

08 juin 2021. URL : http://journals.openedition.org/culturemusees/2845 ; DOI :

https://doi.org/10.4000/culturemusees.2845

\section{Auteur}

\section{Maria Elena Buslacchi}

Université de Gênes, Centre Norbert Elias

Anthropologue, Maria Elena Buslacchi est docteure à l'EHESS - Centre Norbert Elias et à

I'Université de Gênes. Actuellement, elle déroule sa recherche post-doctorale au Lames, Laboratoire méditerranéen de sociologie d'Aix-Marseille Université. Elle a étudié l'impact du titre de Capitale européenne de la culture sur les villes de Gênes (2004), de Marseille (2013) et de Matera (2019), et s'intéresse aux dynamiques de développement des politiques culturelles, au fonctionnement des institutions de la culture ainsi qu'à la réception de l'offre culturelle de la part des publics.

Elle est lauréate du prix 2018 de l'Université franco-italienne et, en 2019, d'une bourse de recherche du ministère de la Culture et de la Communication pour une enquête au sein des musées de Marseille. Ses travaux sont publiés en plusieurs langues.

Courriel : mariaelena.buslacchi[at]gmail.com 


\section{Droits d'auteur}

Culture \& Musées 